\title{
The reablement team's voice: a qualitative study of how an integrated multidisciplinary team experiences participation in reablement
}

This article was published in the following Dove Press journal:

Journal of Multidisciplinary Healthcare

2 November 2016

Number of times this article has been viewed

\author{
Kari Margrete Hjelle ${ }^{1,2}$ \\ Olbjørg Skutle ${ }^{2,3}$ \\ Oddvar Førland 2,4 \\ Herdis Alvsvåg ${ }^{4}$ \\ 'Department of Occupational \\ Therapy, Physiotherapy and \\ Radiography, Faculty of Health and \\ Social Sciences, Bergen University \\ College, Bergen, Norway; ${ }^{2}$ Centre \\ for Care Research Western Norway, \\ Bergen University College, Bergen, \\ Norway; ${ }^{3}$ Department of Health \\ and Social Educators, Faculty of \\ Health and Social Sciences, Bergen \\ University College, Bergen, Norway; \\ ${ }^{4}$ VID Specialized University, Bergen, \\ Norway
}

Background: Reablement is an early and time-limited home-based rehabilitation intervention that emphasizes intensive, goal-oriented, and multidisciplinary assistance for people experiencing functional decline. Few empirical studies to date have examined the experiences of the integrated multidisciplinary teams involved in reablement. Accordingly, the aim of this study was to explore and describe how an integrated multidisciplinary team in Norway experienced participation in reablement.

Methods: An integrated multidisciplinary team consisting of health care professionals with a bachelor's degree (including a physiotherapist, a social educator, occupational therapists, and nurses) and home-based care personnel without a bachelor's degree (auxiliary nurses and nursing assistants) participated in focus group discussions. Qualitative content analysis was used to analyze the resulting data.

Results: Three main themes emerged from the participants' experiences with participating in reablement, including "the older adult's goals are crucial", "a different way of thinking and acting - a shift in work culture", and "a better framework for cooperation and application of professional expertise and judgment". The integrated multidisciplinary team and the older adults collaborated and worked in the same direction to achieve the person's valued goals. The team supported the older adults in performing activities themselves rather than completing tasks for them. To facilitate cooperation and application of professional expertise and judgment, common meeting times and meeting places for communication and supervision were necessary.

Conclusion: Structural factors that promote integrated multidisciplinary professional decisions include providing common meeting times and meeting places as well as sufficient time to apply professional knowledge when supervising and supporting older persons in everyday activities. These findings have implications for practice and suggest future directions for improving health care services. The shift in work culture from static to dynamic service is time consuming and requires politicians, community leaders, and health care systems to allocate the necessary time to support this approach to thinking and working.

Keywords: multidisciplinarity, rehabilitation, goal-setting, framework conditions, work culture, reablement

\section{Introduction}

The elderly population is growing worldwide, and in both Europe and the US an increasing proportion of older people remain in their homes despite declining health. ${ }^{1}$ This trend requires a shift from inpatient care to home-based interventions. New health and social care services need to be developed to promote active user involvement and shared decision making and to focus on supporting and enabling the participation of older adults. ${ }^{1,2}$ Approaching older people by considering their intrinsic capabilities
Correspondence: Kari Margrete Hjelle Centre for Care Research Western Norway, Bergen University College, Postbox 7030, 5020 Bergen, Norway Email kari.hjelle@hib.no 
and the environments in which they live helps ensure that health services are oriented toward the outcomes that are most relevant to these adults' daily lives. ${ }^{2}$

Reablement is a timely service for home-dwelling older people experiencing a decline in health and function that emphasizes their mastery of valued activities and their social participation in society. ${ }^{3}$ In reablement, the home care staff is reorganized from multiple individual care providers into an integrated multidisciplinary team, whose members work together with the person toward his or her goals. ${ }^{4-6}$ The integrated multidisciplinary team consists of occupational therapists, physiotherapists, social educators, nurses, auxiliary nurses and assistants. ${ }^{7}$ Therapists and other health care professionals with a bachelor's degree supervise the homebased care personnel, which includes those with no formal education (ie, assistants), and guide them in encouraging and assisting the person with daily training. ${ }^{7}$

There has been growing international interest in reablement or restorative care as a new intervention for the elderly. ${ }^{8,9}$ The outcomes of reablement in this population have been investigated in three systematic literature reviews. ${ }^{5,10,11}$ These reviews indicate that, to date, there has been inconsistent and limited research evidence demonstrating that reablement improves the individual's physical capacity, performance in personal activities of daily living and health-related quality of life. However, there is emerging evidence from practice that suggests that reablement is a promising intervention. ${ }^{10}$

Currently, few international publications explore health care professionals' experiences of reablement. In New Zealand, King et $\mathrm{al}^{12}$ found that health care workers in a restorative home care intervention had reduced turnover rates and improved job satisfaction compared with those working in a usual home care group. This improvement was found to be a result of the enhanced training, supervision, and flexibility. In England, Rabiee and Glendinning ${ }^{13}$ studied the organization and delivery of home care reablement. The frontline staff in their study agreed that the success or failure of reablement depended on a strong and shared vision of the service, access to specialists' skills and the capacity of the long-term home care service to provide ongoing support to its users. A Norwegian study indicated that mutual recognition and equality between employees in an interdisciplinary reablement team were essential in creating a functioning multidisciplinary team. ${ }^{14} \mathrm{~A}$ qualitative study from Sweden illustrated the value of team members providing "hands-off" support with respect to an individual's sense of autonomy. "Hands-off" support is characterized by health care personnel focusing on the older adults' capabilities and resources and not "doing" too much for them, specifically, personnel support and advice for older persons to perform activities themselves as much as possible. ${ }^{15}$

Furthermore, earlier results revealed the importance of team members maintaining positive attitudes toward one another and developing competence and willingness to learn from each other. Working across professional boundaries was new to team members, and they described it as a stimulating experience. In Norway, Liaaen ${ }^{16}$ studied professional caregivers' experiences with reablement. The results indicated that reablement led to a change in how they worked to ensure the participation of service recipients. Implementing this change required the caregivers to negotiate dilemmas, such as finding a balance between helping and enabling the person. This dilemma was particularly evident when the professionals perceived that the person was struggling to perform an activity. The results further showed that professional caregivers considered it a privilege to enable the service recipients' achievements.

As the literature mentioned earlier shows, empirical studies of health care professionals' experiences of reablement in the community are sparse, and some important gaps remain. In particular, there is little research-based knowledge of health care personnel's experiences working on a reablement team. In addition, little is known about how professionals in reablement manage dilemmas or conflict related to providing "hands-off" support or how members of integrated multidisciplinary teams collaborate and share knowledge with each other. Thus, the aim of this study was to explore and describe how an integrated multidisciplinary team in Norway experiences participation in reablement.

\section{Methods \\ Design}

The study was designed as a qualitative phenomenological hermeneutic study, which is an approach that aims to gather data from the people concerned. ${ }^{17}$ Focus group discussions were conducted to gather descriptive data in the participants' own words. ${ }^{18,19}$ The main advantage of focus groups is the involvement of the participants in the discussion; they share their perspectives, experiences, and opinions. The study was approved by the Norwegian Social Science Data Service (project number 2012/30996).

\section{Reablement intervention}

The current study is part of a larger research program on reablement in home-dwelling adults that includes a 
randomized controlled trial in a rural municipality in Norway with $\sim 15,000$ inhabitants. ${ }^{4,20,21}$ Health care professionals, including occupational therapists, physiotherapists, nurses, and health care personnel such as auxiliary nurses and assistants, were organized into an integrated multidisciplinary team. ${ }^{4}$

The reablement services lasted for a maximum of 3 months for each participant. The intervention was tailored to the older adults' goals, and thus the components of the rehabilitation plan varied, as described in Table $1 .{ }^{4}$ As part of the baseline assessment, the Canadian Occupational Performance Measurement interview was conducted by an occupational therapist or physiotherapist to identify the older adults' valued goals for reablement. These goals were related to the older adults' ability to cope with and participate in important everyday activities at home or in society. Based on these goals, the integrated multidisciplinary team discussed how to tailor the reablement process to support and assist older adults in achieving their goals during the rehabilitation period. Thus, the components of the intervention varied by person and consisted of both general and individual features, as described in Table 1.

\section{Participants and sampling}

Health care personnel working in the integrated multidisciplinary team were organized into two focus groups. One group consisted of six health care professionals with a bachelor's degree including registered nurses, social educators, occupational therapists, and physiotherapists (named the bachelor's degree group; Table 2). The other group consisted of eight home care personnel without formal health care education, including auxiliary nurses and assistants (named the non-bachelor's degree group; Table 3). Thus, the total sample size of the focus group discussions was 14 participants. The participants were all females. Participants in the bachelor's degree group had the main responsibility for the reablement process and supervised those in the non-bachelor's degree group in encouraging and assisting the older person with daily training. ${ }^{4}$ The rationale for creating two groups for the discussion was to ensure that both groups had the opportunity to share their experiences and talk openly without being influenced by the other group. The local reablement project leader recruited the participants. Before the focus groups began, written information on the study was provided and written informed consent was obtained from all participants.

Table I Features of the reablement intervention

\begin{tabular}{|c|c|c|}
\hline \multicolumn{3}{|l|}{ General features } \\
\hline \multicolumn{3}{|c|}{ The rehabilitation period lasted a maximum of 3 months. } \\
\hline \multirow{2}{*}{\multicolumn{3}{|c|}{$\begin{array}{l}\text { An occupational therapist or physiotherapist conducted the COP } \\
\text { interview and developed the rehabilitation plan together with }\end{array}$}} \\
\hline & & \\
\hline \multicolumn{3}{|c|}{ the participant based on the identified activity goals. Thereafter, } \\
\hline \multicolumn{3}{|r|}{ shared goals guided the } \\
\hline \multicolumn{3}{|c|}{ participant throughout the rehabilitation period. } \\
\hline \multirow{2}{*}{\multicolumn{3}{|c|}{$\begin{array}{l}\text { In addition to home care personnel-assisted training, a minimum of } \\
\text { I hour of physiotherapist- and/or occupational therapist-assisted } \\
\text { training was provided each week. }\end{array}$}} \\
\hline & & \\
\hline \multirow{4}{*}{\multicolumn{3}{|c|}{$\begin{array}{l}\text { The treatment involved repetitive training and multiple home visits } \\
\text { from health care personnel, who were present during daily training to } \\
\text { help the older person build confidence and relearn skills. } \\
\text { All health care personnel encouraged the participant's self-management } \\
\text { and self-training. }\end{array}$}} \\
\hline & & \\
\hline & & \\
\hline & & \\
\hline \multirow{2}{*}{\multicolumn{3}{|c|}{$\begin{array}{l}\text { Notes: Reproduced from Tuntland H, Espehaug B, Forland O, Hole AD, Kjerstad } \\
\text { controlled trial. BMC Geriatr. 2014;14:139. @ Tuntland et al. Licensee BioMed Centra } \\
\text { by/4.0/legalcode. }{ }^{20} \\
\text { Abbreviation: COPM, Canadian Occupational Performance Measurement. } \\
\text { Table } 2 \text { Main characteristics of professionals in the bachelor's } \\
\text { group taking part in focus group discussion }\end{array}$}} \\
\hline & & \\
\hline rotession & N/total size & $\mathbf{G e}$ \\
\hline yysiotherapist & I & , $35-40 y$ \\
\hline ccupational therapist & 2 & $\begin{array}{l}\text { Female, } 30-35 \text { years }(I) \text {; } \\
40-45 \text { years }(I)\end{array}$ \\
\hline ial e & I & Female, $40-45$ years \\
\hline Vurse & 2 & $\begin{array}{l}\text { Female, } 50-55 \text { years }(1) \text {; } \\
55-60 \text { years }(I)\end{array}$ \\
\hline otal & 6 & remale \\
\hline
\end{tabular}

\section{Individual features}

Training in daily activities, such as dressing, food preparation, vacuuming, bus transport, visiting friends at a club, or being able to knit, was provided. Adaptations such as advice regarding appropriate assistive technology or adapting the activity itself or the environment to simplify activity performance was provided.

Exercise programs, such as indoor or outdoor walking with or without walking aids, climbing stairs, transferring, and performing exercises to improve strength, balance or fine motor skills, were recommended. The exercises were incorporated into daily routines, and the person was given a manual explaining each of the exercises and was encouraged to train on their own.
Table 3 Main characteristics of home care personnel in the nonbachelor group taking part in focus group discussion

\begin{tabular}{lll}
\hline Profession & N/total size & Gender, age $(\mathbf{n})$ \\
\hline Auxiliary nurses & 2 & $\begin{array}{l}\text { Female, } 40-45 \text { years }(\mathrm{I}) ; \\
45-50 \text { years }(\mathrm{I})\end{array}$ \\
Assistant without formal & 6 & Female, $30-35$ years (2); \\
health care education & & $35-40$ years (2); \\
& & $50-55$ years $(\mathrm{I}) ;$ \\
& & $55-60$ years $(\mathrm{I})$ \\
Total & 8 & Female \\
\hline
\end{tabular}




\section{Data collection method}

The bachelor's degree group was interviewed twice, and the non-bachelor's degree group was interviewed once. Each focus group discussion lasted $\sim 2$ hours including time for refreshments. All interviews were conducted in the participants' community but outside the localities of the reablement service. The interviews were conducted from spring 2013 to spring 2014, 1 year after the reablement service had started in the community. The interview data were digitally recorded and later transcribed verbatim. Two semi-structured interview guides were developed, one for each focus group discussion, according to Kvale and Brinkmann. ${ }^{22}$ Some of the main topics in the interview guides are presented in Boxes 1 and 2. The discussions focused on how the group members experienced working in reablement. Sample questions include the following: "Can you discuss how you have experienced participating in the reablement service? Please discuss the similarities and differences between working in reablement and working in ordinary rehabilitation. Can you discuss how you as the bachelor's degree group collaborate with the non-bachelor's

Box I Topics included in the focus group interview guide of the bachelor's degree group

Please discuss your experiences of participating in the reablement
service.
Please discuss the similarities and differences between working in
reablement and working in ordinary rehabilitation. Please give some
examples.
Can you discuss how you as the bachelor's degree group collaborate
with the non-bachelor degree group?
Please discuss your experiences of working together as an integrative
multidisciplinary team. Please give some examples.
Please discuss how you have supervised the non-bachelor's degree
group.

Box 2 Topics included in the focus group interview guide of the non-bachelor's degree group

Can you discuss your experiences of participating in the reablement
service?
Please discuss the similarities and differences between working in
reablement and working in ordinary rehabilitation. Please give some
examples.
Can you discuss how you as the non-bachelor's degree group
collaborate with the bachelor's degree group?
Please discuss your experiences of working together as an integrative
multidisciplinary team. Please give some examples.
How do you experience the supervision from the professionals of the
bachelor's degree group?
How do you collaborate with the older adult himself/herself?
Could you please discuss your experiences of enabling the older adult
in doing the activities himself/herself.

degree group?" The second author (OS) of this article conducted the discussions and moderated the group sessions, assisted by the first (KMH) and fourth (HA) authors. The first author $(\mathrm{KMH})$ wrote notes during the sessions as well as a summary after each session. ${ }^{18,19}$ These field notes included text and did not use a systematic structure. The researchers ( $\mathrm{KMH}, \mathrm{OS}$, and $\mathrm{HA}$ ) read the transcribed data from the first two focus group discussions to identify statements to further explore and discuss in the second focus group discussion with the bachelor's degree group. The bachelor's degree group was asked to provide descriptive examples of situations in which they had experienced dilemmas or conflicts during collaboration with or supervision of participants in the nonbachelor's degree group. The non-bachelor's degree group was interviewed only once because we determined that we would not gain more information in another interview. Once the group discussions and transcriptions had been completed, all the materials were compiled and treated as a whole by the authors of this article (KMH, OS, OF, and HA).

\section{Data analysis}

The group discussions were analyzed according to and inspired by a phenomenological decontextualization and recontextualization process. ${ }^{23}$ All the authors analyzed the transcribed data separately and together several times to gain a deeper understanding of the text and to ensure the trustworthiness of the analysis. The analysis followed a four-step procedure: Step 1, total impression: from chaos to themes; Step 2, identifying and sorting meaning units: from themes to code groups; Step 3, condensation: from code to meaning; Step 4, synthesizing: from condensation to descriptions and concepts. ${ }^{23}$ In the first step of the analysis, the transcripts were read several times to gain a deeper understanding of the data. To capture the central aspects of the text, relevant concepts were listed in a separate column. We went back and forth between the data and the initial concepts to examine them and ensure that the concepts and emerging codes adhered closely to the empirical data.

In the second step of the analysis, we identified the meaning units, which were text fragments that reflected the information about the participants' experiences of participating in reablement. Then, we began coding by identifying and sorting these meaning units. In this step, we also moved back and forth between the data and the codes to examine the existing codes and add new ones. For example, codes such as "collaboration is motivating", "moving in the same direction", and "help is graded or adapted to the person" emerged. During this decontextualization phase, we reflected 
on the similarities and differences of each code. The codes were discussed continuously by the authors until consensus was reached.

The third step of the analysis involved the systematic abstraction of meaning units within each of the code groups established in the second step of the analysis. The transcripts were read systematically to identify and classify the meaning units into thematic code groups.

In the fourth step of the analysis, the data were recontextualized by developing descriptions and providing stories that fully reflected the original context. This process was outlined in terms of core themes and subthemes and is presented in the findings. To substantiate the core themes and subthemes that were identified, representative text elements from the transcripts are included as quotations in the results. The quotes could be better contextualized using specific data from the participants (ie, female, physiotherapist, married, 36 years old), but we decided not to use quotations to maintain the anonymity of the participants. This decision is further outlined in the methodological considerations.

\section{Results}

As a result of the analyses, three main themes emerged: 1) the older adult's goals are crucial, 2) a different way of thinking and acting - a shift in work culture, and 3) a better framework for cooperation and application of professional expertise and judgment.

\section{The older adult's goals are crucial}

The belief that "the older adult's goals are crucial" was identified as a strong feature of the integrated team's experiences of reablement. Both the focus groups in this study highlighted the importance of the older adult's own goals for the reablement process. Awareness of the older adult's goals guided the professionals both with and without a bachelor's degree in their work and collaboration with the care recipient throughout the rehabilitation period. The analysis identified two subthemes: 1) The key is considering the person's goals and 2) working together toward the same goals is motivating.

\section{The key is considering the person's goals}

The subtheme "the key is considering the person's goals" was experienced and described by the integrated multidisciplinary team as an essential factor of reablement. In the focus group discussion, this subtheme was highlighted as follows:

Actually, this has been a good way to work multidisciplinarily. I think the key is that you actually care about the person's goals. In a way, it is a little more of an overlap of physiotherapy exercises, nursing tasks and other tasks. We have managed to cross over each other's fields.

Both the bachelor's and non-bachelor's degree groups discussed this subtheme and agreed that the older person's goal must be given priority over the health professionals' interests, as illustrated by the following excerpt:

Give the person's goal priority. Yes, that's what guides and drives the rehabilitation process and our daily work. The older adults are much more motivated when they have taken part in the decision making regarding which everyday activities they want to cope with; they get ownership over their own goals and reablement.

Here is another example from the focus group's discussions regarding goal formulation:

The difference between traditional rehabilitation and reablement is actually the goal formulation. The older adults form their own goals. Then, we as health care providers with professional backgrounds come together to discuss how we can practically encourage and support the people to achieve the objectives within the time limit.

\section{Working together toward the same goals is motivating}

The health care personnel on the integrated multidisciplinary team discussed how the person's goals made a difference in their work before and after reablement. In traditional homebased care services and rehabilitation, the person's goals were not the focus and did not guide the intervention, whereas they were the focus of reablement services, as this statement from the focus group discussion illustrates: "We are all working toward the person's goals; that is a driving-force, and it is interesting to work this way".

To support and enable older adults in achieving their goals, the bachelor's degree group stressed that it was necessary and important to have a multidisciplinary team comprising an occupational therapist, physiotherapist, and nurse in collaboration with home-based care personnel. They highlighted that reablement resulted in all the health care personnel collaborating together with the older adult toward the same goals. This process is expressed in the following quote:

Together, we communicate and collaborate with the older person to support and enable him to achieve his goals. That is a way to pull in the same direction. Everybody does it, and we know about each other. That is important. Pulling in the same direction [...] it's a nice picture [...]. Pulling in the same direction is an important element of reablement. 
In summary, the integrated multidisciplinary team perceived the older persons' goals as crucial for allowing team members to collaborate with, support, and enable them. Working together toward the same goals as a team, including the older adult, was motivating for the team members. The participants felt inspired by how the older adults were motivated to train and practice activities when they had decided themselves which everyday activities to address.

\section{A different way of thinking and acting - a shift in work culture}

The health care personnel in our study perceived that their work in reablement required a different way of thinking and acting that represented a shift in work culture. This difference represented a change in perspective from viewing older people as passive recipients because of their disease and illness to considering them as active recipients with resources and the ability to participate in a supported and adapted way. In the focus groups, the health care personnel discussed how home care provided in the community often involved compensatory help that persisted for a long time without being evaluated.

When the reablement service was implemented in the community, some of the health care personnel in the integrative multidisciplinary team perceived it as an additional workload. The reablement approach requires time to change to a different way of thinking and acting, and certain personnel must be a driving force for this change, as expressed in this quote: "It is necessary for the bachelor's group to pull the strings because they grasp this way of thinking and have the overall view." The theme "a different way of thinking and acting - a shift in work culture" encompassed two subthemes: 1) focusing on and searching for the older person's resources and 2) from passive recipient to active participant.

\section{Focusing on and searching for the older person's resources}

Focusing on and searching for the older person's resources were strongly related to the change in perspective from one of a person with disease to one that recognized the older person's resources in performing everyday activities and participating in society. The participants stressed that "now we look more for a healthy patient than only for an ill one". For example, before reablement, the health care personnel decided whether a person needed help with showering and dressing in the morning, and they performed the morning care in a way that made the person passive. Once they became involved in reablement, however, they began to look for the person's ability to perform everyday activities. This was exemplified by the following quote: "We ask ourselves: What are they able to do themselves?" The non-bachelor's degree group discussed how they appreciated that the bachelor's degree group supervised them in identifying the persons' resources and supporting them in performing everyday activities. The non-bachelor's degree group described how being a part of the integrated multidisciplinary team taught them to think differently and changed their approach from performing activities "for" the person to supporting the person in doing activities himself or herself. The shift in work culture also resulted in opportunities to practice professional assessments and expertise in ways that helped older adults take charge of their own life. This was a great experience for team members and helped them develop their professional perspectives and knowledge.

\section{From passive recipient to active participant}

The integrated multidisciplinary team in our study was able to involve the older adult as an active participant in the reablement process. In usual home care, the older person is often a passive recipient of help; however, in reablement, the older person is usually an active participant in physical training and performs everyday activities to achieve his or her goals. Participants in both the bachelor's and non-bachelor's degree groups expressed that it was important to change their way of thinking from "a passive older adult and active personnel to an active older adult and passive personnel". In one focus group, the following was stated:

Before, we were at home and helped him with everything.

He had just received a stoma. However, in this new way of thinking, we cannot do everything for him; instead, we have to supervise him in changing the bag himself. We were in-house and supported him in doing it the right way, and he developed more and more confidence in changing it himself. As health care personnel, we have to remind ourselves to ask, "Is this something the person is able to cope with himself?" However, as personnel, we must gain experience with this way of thinking and working.

The bachelor's degree group was responsible for conducting training that required their specific expertise and for making weekly visits to assess the older adult's performance in priority activities. The professionals then discussed how the service or help could be further organized and supported. Some of the health care personnel in the non-bachelor's degree group were present during the daily training to support the older adult in gaining confidence and relearning skills to 
perform everyday activities. One of the health care personnel offered the following insight:

We don't think in the same way about the patients anymore. We do not think that a person who had a stroke and is receiving home nursing is always in need of having this service. Actually, these patients can stand on their own feet and even arrange their own life themselves.

The integrated multidisciplinary team supported and supervised the older adults in performing activities themselves. However, this required health care personnel to empower older adults to cope with activities on their own and to not do the activities "for" them. One of the participants in the focus groups asked herself and the team, "Are we enabling power? Don't we often ask, 'Does this person really cope with the activities himself?' Do we think we are indispensable?"

Involving the care recipient in the reablement process to cope with everyday activities may increase the older adult's confidence in managing his or her prioritized activities. However, involving the person in performing everyday activities also required the health care personnel to determine how the person would, for example, be able to manage his medication. Otherwise, it was unclear whether the person could be in charge of his own daily life:

\section{We visit a patient once a day to give an injection. The patient was concerned about having to be home at a particular time to get this injection, and this was cumbersome. However, I asked the patient if she wanted to take the responsibility herself. She agreed, and we started the training. She devel- oped confidence in doing it herself.}

In summary, the integrated multidisciplinary team experienced reablement as a different way of thinking and acting and a shift in work culture. The approach to work shifted from static thinking, in which decisions regarding specific tasks and a passive recipient guided the health care personnel's work, to dynamic thinking and working, in which the older adult's goals, resources and active participation guided the work in a different manner. The health care personnel supported the older adults in performing the activities themselves. Dynamic thinking also implies that the person's situation and need for help may change from the beginning to the end of reablement. With reablement, the expectations of the older adults themselves, with input from the health care personnel, determine the goals of the intervention.

\section{A better framework for cooperation and application of professional expertise and judgment}

The participants in the focus group discussions described having formal and informal meeting places and meetings that could be accessed during busy working days. Lunch could serve as an informal meeting, as one member of the bachelor's degree group describes below:

\begin{abstract}
We adjust our time and stop by during lunch and meet many personnel at the same time. That way, we combine the social aspect with the need to share information. I think it's very useful, and it becomes a routine that home care personnel can live with for a long, long time.
\end{abstract}

Some professionals from the bachelor's degree group visited health care personnel in different parts of the health care service in the community and provided information related to how the older adult's reablement was progressing. In addition, the professionals in the bachelor's degree group gave valuable praise to the non-bachelor's degree group and acknowledged their work. However, in the focus group, participants stated that the professional knowledge regarding how to involve and engage older adults in being active participants and achieving their goals was not new; instead, what was new was the framework for professional collaboration and communication. This notion is described in the following example from the discussion:

This knowledge has always been in the community. However, because we didn't have a framework for communication, the service was fragmented. Now, we are actually sitting down together and talking about the older person's goals and are contributing to his achievement of these goals. If we did not have these meetings and meeting places, we would not have reached the goals. It is all about another way to collaborate. There is a significant difference in the system of cooperation and communication. Now there are regular meeting points for health care personnel in addition to telephone contact. It is a very low threshold for talking together.

The participants discussed how having a framework for regular meeting times and meeting places for communication and supervision also meant that the professionals in the bachelor's degree group were able to appreciate the personnel in the non-bachelor's degree group. This appreciation is exemplified by the following quotation: 
It is the well-being of those who practice the job. Having your visits and your information about the progress of the individual and giving a little nod to those who do the tenminute job is important. We all need praise, and it is very good that others also hear that it triggers praise when you do what is being supervised. In this way, you follow up. It is a simple thing to do that can have a pretty large impact. It is so nice getting feedback about how well we are doing our job. It is really gratifying to be part of this reablement service. So there are many pluses in the working day.

In the focus group discussions, several of the participants challenged themselves regarding the particular knowledge and skills their own profession contributed. These discussions represented a new and broader professional perspective and view of the older persons' situation, as shown by the following remark:

I think I see the older adult's whole situation. However, then I talk to a nurse or an occupational therapist, and then I say to myself: Oh, no, I have not been thinking of that. After this talk, I have a broader view of the situation.

In summary, the better framework for cooperating and applying professional expertise and judgment included a framework for working toward common goals, providing times and places for common meetings for professional collaboration and communicating with and supervising all health care personnel. To ensure adequate rehabilitation, it is necessary for personnel to discuss the progress in older persons' training programs and their ability to perform everyday activities. This framework provided an improved opportunity to apply professional knowledge and judgment.

\section{Discussion}

One of the main findings in our study was that the older adult's goals guided the reablement process and the integrative multidisciplinary team's work. All the health care personnel worked together with the older person to achieve his or her valued goals. This is in-line with Randström et $\mathrm{al}^{15}$ and a review by Tessier et al, ${ }^{5}$ which emphasize the importance of professionals' sensitivity to the older person's goals for rehabilitation. A study by Hjelle et $\mathrm{a}^{21}$ highlighted that an integrated multidisciplinary approach focusing on the older adult's goals was crucial to encouraging the care recipient to practice and achieve his/her goals. Many international studies in recent decades have investigated how professionals work together with the person in the rehabilitation context. These studies discuss shared goals, patient-centered care, collaboration and teamwork. ${ }^{10,14,24-26}$ However, our findings indicate that the cooperation that occurred between the bachelor's and non-bachelor's degree groups was new and essential for all health care providers to collaborate with older adults to achieve their goals for reablement.

A different way of thinking, involving a shift in work culture from static to dynamic service, was another clear feature of the integrated multidisciplinary team's experiences with reablement. The health care providers acted differently when collaborating with the service recipients to encourage them to become more active and perform tasks themselves. The team developed a common understanding of what was expected of the service recipients and of the staff to ensure that the staff members did not help too much. As in our study, participants in studies by Liaaen ${ }^{16}$ and Randström et $a l^{15}$ stated that the professional care provider was more of an enabler and facilitator, helping older adults perform everyday activities and participate, rather than a helper who performed activities for the older adults. Others have noted that providing nonintrusive, "hands-off" or "stand back" support, rather than lending a helping hand, is a central part of home rehabilitation. ${ }^{5,9,11}$ The participants in these studies expressed that they had to think and act differently and revise some of their tendencies from "doing for" to "letting them do as much as possible themselves". This shift was confirmed in a study by Hjelle et al, ${ }^{21}$ in which health care personnel encouraged and supervised older people's efforts to exercise and perform activities while some personnel were at their home. However, Liaaen ${ }^{16}$ found that implementing this different approach required negotiating dilemmas such as finding a balance between helping and enabling the person. This dilemma was particularly evident when professionals knew that the person was struggling to perform activities.

The participants in our study highlighted the fact that the professional knowledge regarding stimulating and supporting care recipients to perform everyday activities themselves was well known and not new to reablement service. They asserted that all health care personnel are taught to search for the care recipient's resources and that this is the foundation of the intervention. However, prior to reablement, the professionals in the health care service were not able to apply their professional expertise and judgment regarding "doing with" and not "doing for" the patient because of time restrictions in traditional home care service. The reablement approach provided opportunities to apply their professional assessment and expertise in ways that supported and enabled the older adult's ability to be in charge of his or her own life. A better framework for cooperation and application of professional 
knowledge and judgment enabled the professionals to work in a goal-oriented and person-centered manner. The quality of work was based on having this framework, as it provided time to demonstrate professional competence. In our study, the reablement service was not limited to home visits with predetermined minutes. This lack of time constraint gave the professionals the opportunity to motivate and encourage the care recipient to be an active participant in the reablement services. For older adults to be active participants in their own care, health care providers must have enough time to wait for the person to perform everyday activities. In this approach, the integrative multidisciplinary team was able to use the knowledge from Aristotle ${ }^{27}$ called "phronesis", that is, knowledge in terms of discretion and good judgment in action. Despite the time needed, some of our participants expressed that this way of working was in-line with their professional competence and allowed them to use their knowledge and reflections in action. ${ }^{28}$ Similarly, King et al ${ }^{12}$ highlights that a restorative philosophy enables the caregivers to interact and support the older person in a meaningful way, and they describe this as holistic as opposed to a service with restrictions. In their study, Tessier et $\mathrm{al}^{5}$ confirmed that home care services should focus less on time and tasks and more on support for the individual to achieve his or her goals.

In our study, the participants noted that what was new about the reablement approach was its improved framework, as it allowed all health care personnel to apply their knowledge together with the older adult and work in the same direction, ie, toward the older adult's goals. Reablement services require a common physical location where all team members can meet to exchange important information, share knowledge, provide and receive supervision, and discuss and plan their work together. This improved framework was necessary and facilitated collaboration. Randström et $\mathrm{al}^{15}$ also noted the value of communication and of planning and effectively transferring information across all health care personnel and emphasized that having a common physical location where all team members could meet and plan their work facilitated collaboration.

Communication between all health care personnel involved in reablement is necessary to support and enable older persons to achieve their goals. Working in an integrated multidisciplinary team also implies respect for others' competence and knowledge. This finding is in-line with a study by Moe and Brataas, ${ }^{14}$ who noted the importance of team members maintaining positive attitudes toward each other. However, our study findings emphasized that the opportunity for team members to supervise and evaluate each other required a framework for organized meetings in which knowledge sharing could occur. The participants in our study highlighted the significance of a better framework for communication and dialog among all health care personnel. Powell et $\mathrm{al}^{29}$ argued that the prerequisites for a successful implementation and improvement of an intervention or service are that all health care personnel involved are engaged in and take ownership of the new knowledge or service. The service must be adapted to the local environment and community. Furthermore, the leadership of the service must be supportive and must participate in implementing the new service. These requirements have implications for the health care system, community leaders, and politicians.

\section{Methodological considerations}

The strength of this study, which applied a qualitative approach to examine integrated multidisciplinary teams' experiences with participating in reablement, is that it provides valuable insight from stakeholders regarding the reablement process. When researching and developing reablement services, it is important for the voices of those involved in the rehabilitation approach to be heard. In qualitative studies, the results are evaluated on their credibility. ${ }^{22,23}$ The credibility in this study resulted from the fact that the participants in the focus groups represented all health care providers in the reablement service and thus provided varied perspectives on the phenomenon studied. All the participants in the focus group discussions participated with engagement and enthusiasm. The reason for creating two focus groups, the bachelor's and non-bachelor's degree groups, was to ensure that both groups had the opportunity to talk openly and not be influenced by the other group. Mixed groups could have influenced the results, as participants may not have shared and fully discussed their opinions and experiences. However, in further research, it could be interesting to have one focus group discussion with both groups together to reflect and discuss the integrated multidisciplinary team's experiences as a whole.

The local reablement project leader recruited the participants and did not participate in the focus group discussion. However, in the second focus group discussion, one of the participants was a project leader. We believe that this did not influence the discussion or the results of our research. She did not affect the discussion or influence the participants in expressing their opinions and experiences, and as she had participated in the first focus group discussion, we did not want to exclude her from the second one. 
Participants in the non-bachelor's degree group provided valuable insight into their experiences in reablement services; however, this group was interviewed only once. The participants expressed their experiences rather concisely in the first interview, and we believed that we would not obtain more information from another interview. According to Malterud, ${ }^{23}$ researchers have to consider the intersubjectivity and consistency of the data according to the aims of the study. Our study intends neither to compare the results of the two group discussions nor to provide a complete picture of the experiences of the integrated multidisciplinary team's participation in reablement.

Another strength of our study is that all the authors analyzed the transcribed data separately and together several times to gain a deeper understanding of the data and to ensure the trustworthiness of the analysis. Credibility was further established by using representative text from the transcripts as quotations in the reporting of the results. ${ }^{22,23}$ The quotes would have been better contextualized with specific data from the participants (ie, female, physiotherapist, married, 36 years old), but we determined that including specific participant characteristics was not recommended as it compromised the anonymity of the participants. The participants were recruited from a rather small community, and they may have been recognized. In the sampling, only one or two persons represented their specific professions.

One limitation of our study is that the participants were recruited from one reablement project that had funding. ${ }^{4}$ Funding implied a better framework that was not as limited as those found in ordinary rehabilitation settings. This may have affected our participants' enthusiasm and their positive experiences with reablement. However, they reflected on and discussed both positive and less positive perspectives of the reablement service.

Moreover, the empirical data are from two groups of health care professionals with a total of three focus group discussions, which may have added further bias to a sample with a limited number of participants. As in most qualitative studies, the number of participants was limited. This smaller sample size means that the study participants do not necessarily represent other health care personnel engaged in reablement services. Nonetheless, the study provided indepth insight into one reablement team that could be related to similar situations or teams. For example, our findings regarding the shift in work culture from the patient as a passive recipient to the patient as an active participant, as well as the provision of a better framework for professional expertise and judgment, could inspire other reablement teams. These findings may also motivate home care services, although many older adults living at home are unable to have the same goals for independence as older adults participating in reablement.

\section{Conclusion and implications}

Reablement services require sufficient time for integrated multidisciplinary teams to apply their professional knowledge when supervising and supporting older persons' efforts to perform everyday activities themselves. This implies that politicians, community leaders, and health care systems support a framework for implementing this approach. An improved framework for integrated multidisciplinary team work and the use of professional expertise and judgment includes factors such as meeting times and meeting places necessary for professional collaboration and decision making. This type of framework also requires that the integrated multidisciplinary team has enough time to apply their professional knowledge when supervising and supporting the older person in performing everyday activities himself/herself. The shift in work culture from static to dynamic service is time consuming, and managers and policy makers have to consider this added time when developing strategic plans. Future research could explore the integrated multidisciplinary team's experiences after the completion of the project, when the service is part of the routine health care services in the community. In addition, research could further explore how the bachelor's degree group experienced their professional role when they delegated some of their traditional tasks to health care personnel without a bachelor's degree.

\section{Acknowledgments}

$\mathrm{KMH}, \mathrm{OS}$, and HA are sponsored by Regional Research Funds Western Norway, grant number 229759. We thank all the participants who willingly shared their experiences with reablement with us. We also thank the local project leaders for recruiting the participants for our research.

\section{Disclosure}

The authors report no conflicts of interest in this work.

\section{References}

1. UNECE. Innovative and Empowering Strategies for Care. Policy Brief Age. Vol. 15. Geneva: UNECE; 2015.

2. WHO. World Report on Ageing and Health. Geneva: WHO; 2015.

3. Rabiee P, Glendinning C. Organisation and delivery of home care re-ablement: what makes a difference? Health Soc Care Community. 2011;19(5):495-503.

4. Tuntland H, Aaslund MK, Espehaug B, Førland O, Kjeken I. Reablement in community-dwelling older adults: a randomised controlled trial. $B M C$ Geriatr. 2015;15(1):1-11

5. Tessier A, Beaulieu M, Mcginn C, Latulippe R. Effectiveness of reablement: a systematic review. Healthc Policy. 2016;11(4):49-59. 
6. Tinetti ME, Charpentier P, Gottschalk M, Baker DI. Effect of a restorative model of posthospital home care on hospital readmissions. $\mathrm{J} \mathrm{Am}$ Geriatr Soc. 2012;60(8):1521-1526.

7. Kjerstad E, Tuntland HK. Reablement in community-dwelling older adults: a cost-effectiveness analysis alongside a randomized controlled trial. Health Econ Rev. 2016;6(1):1-10.

8. Cochrane A, McGilloway S, Furlong M, Molloy David W, Stevenson M, Donnelly M. Home-care 're-ablement' services for maintaining and improving older adults' functional independence. Cochrane Database Syst Rev. 2013;11:CD010825.

9. Aspinal F, Glasby J, Rostgaard T, Tuntland H, Westendorp RG. Reablement: supporting older people towards independence. Age Ageing. Epub 2016 Sep 19.

10. Whitehead PJ, Worthington EJ, Parry RH, Walker MF, Drummond AE. Interventions to reduce dependency in personal activities of daily living in community dwelling adults who use homecare services: a systematic review. Clin Rehabil. 2015;29(11):1064-1076.

11. Legg L, Gladman J, Drummond A, Davidson A. A systematic review of the evidence on home care reablement services. Clin Rehabil. 2016;30(8):741-749.

12. King AI, Parsons M, Robinson E. A restorative home care intervention in New Zealand: perceptions of paid caregivers. Health Soc Care Community. 2012;20(1):70-79.

13. Rabiee P, Glendinning C. Organisation and delivery of home care re-ablement: what makes a difference? Health Soc Care Community. 2011;19(5):495-503.

14. Moe A, Brataas HV. Interdisciplinary collaboration experiences in creating an everyday rehabilitation model: a pilot study. Journal of Multidisciplinary Healthcare. 2016;9:173.

15. Randström KB, Wengler Y, Asplund K, Svedlund M. Working with 'hands-off' support: a qualitative study of multidisciplinary teams' experiences of home rehabilitation for older people. Int J Older People Nurs. 2014;9(1):25-33.

16. Liaaen JMA. Professional carers' experience of working with reablement [master]. Trondheim: Fakultet for helse- og sosialvitenskap, Høgskolen i Sør-Trøndelag; 2015.
17. Giorgi A. An application of phenomenological method in psychology. In: Giorgi C, Fischer C, Murray E, editors. Duquesne Studies in Phenomenological Psychology. Pittsburgh: Duquesne University; 1975: 82-103.

18. Krueger RA. Moderating Focus Groups. Thousand Oaks, CA: Sage; 1998.

19. Barbour RS, Kitzinger J, editors. Developing Focus Group Research. Politics, Theory and Practice. London: SAGE Publications Ltd; 1999.

20. Tuntland H, Espehaug B, Forland O, Hole AD, Kjerstad E, Kjeken I. Reablement in community-dwelling adults: study protocol for a randomised controlled trial. BMC Geriatr. 2014;14:139.

21. Hjelle KM, Tuntland H, Førland O, Alvsvåg H. Driving forces for home-based reablement; a qualitative study of older adults' experiences. Health Soc Care Community. 2016. Early view.

22. Kvale S, Brinkmann S. Interviews: Learning the Craft of Qualitative Research Interviewing. 2nd ed. Los Angeles: Sage; 2009.

23. Malterud K. Systematic text condensation: a strategy for qualitative analysis. Scand J Public Health. 2012;40(8):795-805.

24. van Dongen JJJ, Lenzen SA, van Bokhoven MA, Daniëls R, van der Weijden T, Beurskens A. Interprofessional collaboration regarding patients' care plans in primary care: a focus group study into influential factors. BMC Fam Pract. 2016;17(1):1.

25. Bodenheimer T, Handley MA. Goal-setting for behavior change in primary care: an exploration and status report. Patient Educ Couns. 2009;76(2):174-180.

26. Scobbie L, Dixon D, Wyke S. Goal setting and action planning in the rehabilitation setting: development of a theoretically informed practice framework. Clin Rehabil. 2011;25(5):468-482.

27. Aristotle. Nicomachean Ethics. Indianapolis: Hackett Publisher 1985.

28. Schön DA. The Reflective Practitioner: How Professionals Think in Action. New York: Basic Books; 1983.

29. Powell AE, Rushmer RK, Davies HTO. A Systematic Narrative Review of Quality Improvement Models in Health Care. Edinburgh: NHS Scotland; 2009.
Journal of Multidisciplinary Healthcare

\section{Publish your work in this journal}

The Journal of Multidisciplinary Healthcare is an international, peerreviewed open-access journal that aims to represent and publish research in healthcare areas delivered by practitioners of different disciplines. This includes studies and reviews conducted by multidisciplinary teams as well as research which evaluates the results or conduct of such teams or health

\section{Dovepress}

care processes in general. The journal covers a very wide range of areas and welcomes submissions from practitioners at all levels, from all over the world The manuscript management system is completely online and includes a very quick and fair peer-review system. Visit http://www.dovepress.com/ testimonials.php to read real quotes from published authors. 